\title{
Restoration of Noisy Ultrasound Image with Rectilinear Effect using Curvelet
}

\author{
Komal Juneja \\ Manav rachna international University \\ Faridabad, India
}

\author{
Akash Tayal \\ Indira Gandhi Institute of Technology, GGSIPU \\ New Delhi, India
}

\begin{abstract}
Ultrasound Imaging is primary modality in the diagnosis of many diseases. Compared to other imaging techniques ultrasound imaging owes its great popularity to the fact that it is safe and noninvasive procedure for visualizing the heart, vasculature, abdomen, fetal monitoring etc. Ultrasound images are degraded by intrinsic artifacts called speckle which is result of constructive and destructive coherent summation of ultrasound echoes. In ultrasound imaging if a relative motion exist (subject moves from its position) during imaging the recorded image will be blurred. This effect can be expressed by an impulse response in received echo pulses and respective image restoration is made possible by a post recording process. In this paper we proposed the method to remove the blurring and additive speckle noise is removed by curvelet transform domain which uses cycle spinning.
\end{abstract}

\section{Keywords}

Echo Pulses, Curvelet transform, Cycle Spinning

\section{INTRODUCTION}

In medical image processing image denosing has become a very essential exercise all through the diagnose. Medical images are usually corrupted by noise in its acquisition and transmission. The main objective of image denoisng technique is necessary to remove such noises while retaining as much as possible the important signal feature. The imaging process in ultrasound imaging is based on the principle of reflection of sound waves. The tissue is insonified with sound pulses with a fixed frequency. Subsequently, the reflections that are generated during wave propagation in the tissue due to difference in tissue densities. These reflections are registered as a function of time. The time elapsed between the emission of a pulse and the reception of its echoes reveals the depth of the reflecting objects or tissues interfaces. The intensity of the echo waves yields information on the acoustics properties of the object. These received signals are used to construct an image [1].Ultrasound imaging is widely used medical imaging procedure because it is economical, comparatively safe, transferrable and adaptable though, one of its main shortcomings is the poor quality of images which are effected by speckle noise. The existence of speckle is unattractive since it disgrace image quality and it effects the tests of individual interpretation and diagnosis. Although ultrasound imaging has reached a high level of technical sophistication, patient motion due to any reason is a fundamental issue that has to be addressed in interpreting ultrasound images. The characterization of the image degradation by a Point Spread Function (PSF), so that the degraded image can be expressed by the convolution of the ideal or original image with the respective PSF. A general description of blurring due to the linear motion is proposed by [2] direct delta function properties. The emitted ultrasound pulse is the impulse function of the system. Correspondingly, the received echo pulses can be considered as the impulse response of the biological system. When it represents the output of the ultrasound system during interrogation of the ideal point target, the echo pulses is also known as the System's Point Spread Function. In order to achieve image restoration and evaluation one must know the nature of the degradation as well its effect in order to operate the image to compensate the image for degradation. In Linear smear restoration where a PSF (a rectangular or window function for the case of uniform velocity) shows the application of convolution operation. The dynamic effects of body such as respiration, eyes blinking are neglected. In most cases ultrasound images are corrupted by speckle multiplicative noise. The goal of de-noising is to remove noise and/or spurious details from a given possibly corrupted digital image while maintaining essential features such as edges and it is very difficult to suggest a robust method which works equally well in presence of different types of noise. Many algorithms have been proposed for noise removal, but the recent trend to noise removal is the use of curvelet transforms with cycle spinning technique (CTCS) [3].

\subsection{Curvelets in Image Processing}

In 2002, the first-generation curvelet transform was applied for the first time to image denoising by Starck et al. [4], and by Candes and Guo[5]. The applications of the first-generation curvelets were extended to image contrast enhancement [6] and astronomical image representation [7] in 2003, and to fusion of satellite images [8] in 2005. After the effective second generation curvelet transform [9] had been proposed in 2004, the applications of curvelets increased very fast in many fields involving image/video presentation, denoising and classification. For instance, Ma et al. applied the secondgeneration curvelets for motion estimation and video tracking of geophysical flows [10] and deblurring [11]. Ma and Plonka presented two different models for image denoising by combining the discrete curvelet transform with nonlinear diffusion schemes. In the first model [12], a curvelet shrinkage is applied to the noisy data, and the result is further processed by a projected total variation diffusion in order suppress pseudoGibbs artifacts. In the second model [13], a nonlinear reactiondiffusion equation is applied, where curvelet shrinkage is used for regularization of the diffusion process Starck et al. [14, 15] applied curvelets for morphological component analysis. Recently, B. Zhang et al. [16] used curvelets for Poisson noise removal in comparison with wavelets and ridgelets. In [17], C. Zhang et al. successively applied the multiscale curvelet 
transform to multipurpose watermarking for content authentication and copyright verification. Jiang et al. [18] considered structure and texture image inpainting with the help of an iterative curvelet thresholding method. Tessens et al. [19] proposed a new context adaptive image denoising by modeling of curvelet domain statistics. By performing an inter-sub-band statistical analysis of curvelet coefficients, one can distinguish between two classes of coffecients: those that represent useful image content and those dominated by noise. Using a prior model based on marginal statistics, an appropriate local spatial activity indicator for curvelets has been developed that is found to be very useful for image denoising, see [19]. Geback et al. [20] applied the curvelets for edge detection in microscopy images.

\subsection{Ultrasound Imaging}

Sound generated above the human hearing range (typically 20 $\mathrm{kHz}$ ) is called ultrasound. Ultrasound imaging, also called ultrasound scanning or sonography, involves exposing part of the body to high-frequency sound waves to produce pictures of the inside of the body. Ultrasound exams do not use ionizing radiation (as used in x-rays). Because ultrasound images are captured in real-time, they can show the structure and movement of the body's internal organs, as well as blood flowing through blood vessels. In medicine, ultrasound is used to detect changes in appearance of organs, tissues, and vessels or detect abnormal masses, such as tumors. In an ultrasound examination, a transducer both sends the sound waves and records the echoing waves. When the transducer is pressed against the skin, it directs small pulses of inaudible, high-frequency sound waves into the body. As the sound waves bounce off of internal organs, fluids and tissues, the sensitive microphone in the transducer records tiny changes in the sound's pitch and direction. These signature waves are instantly measured and displayed by a computer, which in turn creates a real-time picture on the monitor.

\section{ANALYSIS}

\subsection{Restoration of Blurring due to Rectilinear Motion}

The Radio Frequency (RF) echo graphic signal usually modeled as the convolution of ultrasound point spread function (PSF) $h$ $(\mathrm{x}, \mathrm{y}, \mathrm{z})$ and the tissue scattering function $\mathrm{t}(\mathrm{x}, \mathrm{y}, \mathrm{z})$ the real plane [4].

$$
s_{r}(x, y, z)=h(x, y, z) * t(x, y, z) .
$$

Where * denotes the convolution operation. The tissue scattering function represents the tissue properties along the propagation direction of ultrasound pulse. A simple tissue model is given by.

$$
t(x, y, z)=\sum_{n} g_{n} \delta\left(x-x_{n}\right)\left(y-y_{n}\right)\left(z-z_{n}\right)
$$

Where $\delta(\cdot)$ is a Dirac function, $\left(x_{n}, y_{n}, z_{n}\right)$ is the center of each inhomogeneity and $g_{n}$ is the echogenecity of the each scatterer of tissue. To model a 2-D ultrasound image, PSF is considered is to be separable, $h(x, y, z)=h(x, y) * h_{z}(z)$. Therefore a 2-D slice is obtained as [21]

$s_{r}(x, y)=h(x, y) * t(x, y)$ and $h(x, y)$ is the impulse response in received echo pulses (point spread function due to motion). Image can be described by a two dimensional function $\mathrm{t}(\mathrm{x}, \mathrm{y})$ when there is a linear motion in one direction at angle $\theta$ with respect to the $\mathrm{x}$ axis. The impulse response in received echo pulse is given by:

$h_{2 D}(x, y)=h\left(x^{\prime}\right) \delta\left(y^{\prime}\right)$.

Where

$\left[\begin{array}{l}x^{\prime} \\ y^{\prime}\end{array}\right]=\left[\begin{array}{cc}\cos Q & \sin Q \\ -\sin Q & \cos Q\end{array}\right]\left[\begin{array}{l}x \\ y\end{array}\right]$.

we consider the motion across the $\mathrm{x}$-axis

$h_{2 D}(x, y)=h(x) \delta(y)$.

$s_{r}(x)=t(x, y) * *(h(x) \delta(y))$.

$s_{r}(x)=t(x) * h(x)$.

Only one direction has to be considered.

$$
\begin{aligned}
& h(x)=1 / T \int_{o}^{T} d t \delta(x-\Delta x[t]) . \\
& h(x)=\frac{1}{T} \int_{-\infty}^{\infty} d t \delta(x-\Delta x[t]) \text { rect }\left(\frac{t-T / 2}{T}\right) .
\end{aligned}
$$

$\mathrm{T}$ is register time, $\Delta \mathrm{x}[\mathrm{t}]$ is the displacement between the changed position of patient from its actual position. $\delta(\mu)$ is the Dirac delta function.

$s_{r}(x)=t(x)^{*} h(x) . s_{r}(x)$ is blurred image and deblurring is done by [22] again deconvolving the convoluted image with the PSF.

\subsection{Curvelet Transform (CT)}

The CT developed by candes \& Donho is a multiscale directional transform which allows an almost optimal non adaptive sparse representation of objects with edges. Most natural images/signals exhibit line like edges, i.e., discontinuities across curves (line or curve singularities). . In order to overcome the missing directional selectivity of conventional two-dimensional discrete wavelet transform, a multiresolution geometric analysis (MGA), named CT, was proposed [23, 24, 25, 26, 27]. In the two dimensional (2D) case, the CT allows an almost optimal sparse representation of objects with singularities along smooth curves. For a smooth object $f$ 
with discontinuities along $\mathrm{C}^{2}$-continuous curves, the best $\mathrm{N}$ term approximation $f_{N}$ that is a linear combination of only $\mathrm{N}$ elements of the curvelet frame obeys $\left\|f-\widetilde{f}_{N}\right\|_{2}{ }_{2} \leq C N^{2}(\log N)^{3}$ ,while for wavelets the decay rate is only $N^{-1}$ the CT uses angled polar wedges or angled trapezoid windows in frequency domain. Wavelet and other frequency transforms are widely used for denoising, but they suffer from shift and rotation sensitivity, as well as they are poor in directionality. CT are more suitable for detection of directionality properties as they provide optimally sparse representations of objects which display curve punctuated smoothness - smoothness except for discontinuity along a general curve with bounded curvature. The idea of the curvelet construction presented here is directly inspired from the work of Donoho and Cand $\mu$ es [28]. Curvelets constitute a relatively new family of frames that are designed to represent edges and other singularities, along curves, much more efficiently than the traditional wavelet transforms. The idea of curvelet [28] is to represent a curve as a superposition of functions of various lengths and widths obeying the scaling law width $\approx$ length $^{2}$.

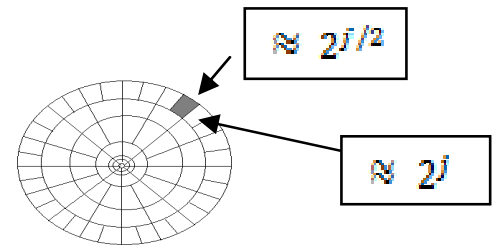

Fig.1: Curvelet tiling of space. The figure represents the induced tiling of the frequency plane. in fourier space, curvelets are supported near a "parabolic" wedge, and the shaded area represents such a generic wedge

\subsection{Digital Curvelet Transform (DCT)}

$\mathrm{CT}$ is to restore sparsity by reducing redundancy across scales. CT can sparsely characterize the high-dimensional signals which have lines, curves or hyper plane singularities and the approximation efficiency is one magnitude order higher than wavelet transform [29].

The DCT of a continuum function $\mathrm{f}\left(x_{1}, x_{2}\right)$ makes use of a dyadic scales, and a bank of filters $\left(P_{0} f, \Delta_{1} f, \Delta_{2} f \ldots \ldots.\right)$ with the property that the pass band filter $\Delta$ is concentrated near the frequencies $\left[2^{2 s}, 2^{2 s+2}\right]$ The curvelet decomposition is the sequence of following steps:

- Subband decomposition: The object $f$ is decomposed into subbands: $f \rightarrow\left(P_{0} f, \Delta_{1} f, \Delta_{2} f \ldots \ldots\right)$

- Smooth Partitioning: Each subband is smoothly windowed into "squares" of an appropriate scale (of side length $\left.\approx 2^{-s}\right): \Delta_{s} f \rightarrow\left(w_{Q} \Delta_{s} f\right)_{Q \in Q_{s}}$

- Renormalization: Each resulting square is renormalized to unit scale

$g_{Q}=\left(T_{Q}\right)^{-1}\left(w_{Q} \Delta_{s} f\right), \quad Q \in Q_{s}$
- Ridgelet analysis : Each square is analyzed via discrete ridgelet transform

\section{DENOSING USING CURVELETS}

\subsection{Speckle Noise in Ultrasound Image}

Speckle noise-the image with this type of noise display granular pattern due to dispersion of electromagnetic waves caused by transducer. When the waves reflected on the rough texture make an impact on said texture they create interferences which cause noise in the registered images. This noise is very harmful since it limits the detection of injuries especially in the low contrast image. Within each resolution cell a number of elementary scatters reflect the incident wave towards the sensor. The backscattered coherent waves with different phases undergo a constructive and destructive interference in a random manner. The acquired image is thus corrupted by a random granular pattern called speckle that delays the interpretation of image content. Speckle noise has the characteristic of multiplicative noise [34]. Speckle is a random, deterministic, interference pattern in an image formed with coherent radiation of a medium containing many sub-resolution scatterers. The texture of the observed speckle pattern does not correspond to underlying structure. The local brightness of the speckle pattern, however, does reflect the local echogenicity of the underlying scatterers. Speckle has a negative impact on ultrasound imaging. Bamber and Daft show a reduction of lesion detectability of approximately a factor of eight due to the presence of speckle in the image [35]. The following model is considered as good model for images with speckle noise

$f(x, y)=g(x, y) \cdot \eta_{m}(x, y)+\eta_{a}(x, y)$

$f(x, y)$ real noisy image, $g(x, y)$ unknown noise free image $\eta_{m}(x, y)$ and $\eta_{a}(x, y)$ multiplicative and additive noise functions since additive noise is considered to be lower than multiplicative noise, loupas et.al (1989) proposed the following signal dependent noise model for speckle specification in ultrasound images.

\subsection{Hard-Threshold Denoising using CT}

The fundamental principle of hard-threshold denoising using CT is consistence with hard-threshold denoising using wavelet transform [30] [31]. The noisy image with additive speckle is defined as:

$$
f(m, n)=f_{0}(m, n)+N(m, n)
$$

Where $f_{0}$ is "clean" image, $N$ is speckle noise, with suitable value of variance Since the DCT if not norm preserving, the variance of each curvelet coefficient depends on its index. The operator of CT is defined as $T, f^{C T}=T f$ denotes curvelet coefficients of noisy image $f, \gamma$ indicates index, $\hat{f}_{\gamma}{ }^{c T}$ curvelet coefficients after hard-threshold processing, and thus denoising function is defined as:

$$
f_{\gamma}^{C T}=\left[\begin{array}{c}
f_{\gamma}^{C T},\left|f_{\gamma}^{C T}\right| \geq k \sigma \sigma_{\lambda} \\
0,\left|f_{\gamma}^{C T}\right|<k \sigma_{\gamma}
\end{array}\right.
$$

Where $\sigma^{2}$ variance of noise is after $\mathrm{CT} \mathrm{k}$ is a sub band dependent value estimated is few known images and letting $\mathrm{k}$ variate. Hard-threshold denoising method can keep edge 
information perfectly, but visual distortion appears in the reconstructed images, such as ringing effect and false Gibbs phenomenon.

\subsection{Curvelet Denoising with Cycle Spinning}

In order to suppress false Gibbs phenomenon due to the lack of translation-invariance with CT, Coifman and Donoho proposed cycle spinning approach [32] [33]. In this paper, we propose image denoising method combining curvelet transform with cycle spinning (CTCS). The implementation procedure is as follows:

(1) Cycle spin noisy image with the number of translations (per dimension) is 1 and iterate 7 times.

(2) Apply (CT) to translated image and obtain curvelet coefficients $f_{\gamma}^{C T}$ at all scales and directions.

(3) Use hard-thresholding to coefficients $f_{\gamma}^{C T}$ obtaining $\hat{f}_{\gamma}^{C T}$ and at the finest scale $k=2.5$ and at all scales except the finest scale $k=2.2$

(4) Apply inverse CT to $\hat{f}_{\gamma}^{C T}$ and obtain denoised image $\hat{f}$.

(5) Apply to inverse cycle spinning to $\hat{f}$.After 7 times iterations, compute the mean value of $\hat{f}$ to get the filtered result.

\section{EXPERIMENTAL RESULT}

For our experiments, we selected some $256 \times 256,512 \times 512$ and $1024 \times 1024$ ultrasound images and the images were blurred due to movement of subject with linear velocity of $10 \mathrm{~m} / \mathrm{sec}$ in $\mathrm{x}$ direction for $2 \mathrm{sec}$ and corrupted with additive speckle noise of different variance and the denoising method was applied. The degraded image was restored by deconvolving the degraded image with respective PSF and the noisy image was filtered using hard thresholding of the transform coefficients and another method using cycle spinning technique. The performance metric used are mean square error (MSE) and peak signal to noise ratio (PSNR).

MSE and PSNR is given by which requires two $M \times N$ gray scale images, the original image $I$ and the denoised image $\hat{I}$, is defined as $M S E=\frac{1}{M N} \sum_{i=0}^{m-1} \sum_{j=0}^{n-1}|I[m, n]-\hat{I}[m, n]|^{2}$

PSNR is inversely proportional to root mean square value (RMSE), its units are in decibels (dB) and is formally defined by

$P S N R=20 \log _{10}\left[\frac{255}{R M S E}\right](d b)$

where 255 is the maximum pixel value for an 8 bits/pixel grayscale image.Table 1 and 2 shows PSNR results of reconstructed images denoised by different methods, as objective evaluation criterion of denoising performance. It can be seen: (1) The image denoised by CTCS is better than the one denoised by CT, illustrating the efficiency of cycle spinning applying to image denoising. (2) To texture rich ultrasound image of $256 \times 256$, 512 x 512 and 1024 x 1024, CSCT increases PSNR $1.7 \mathrm{db}, 8 \mathrm{db}$ and $2.3 \mathrm{~dB}$ on average compared to CT. Fig2 shows the comparison chart of PSNR v/s variance of different denoising method of image size $256 \times 256,512 \times 512$ and 1024 x 1024 .

Fig 3 shows the simulation result of ultrasound image of size $256 \times 256$.
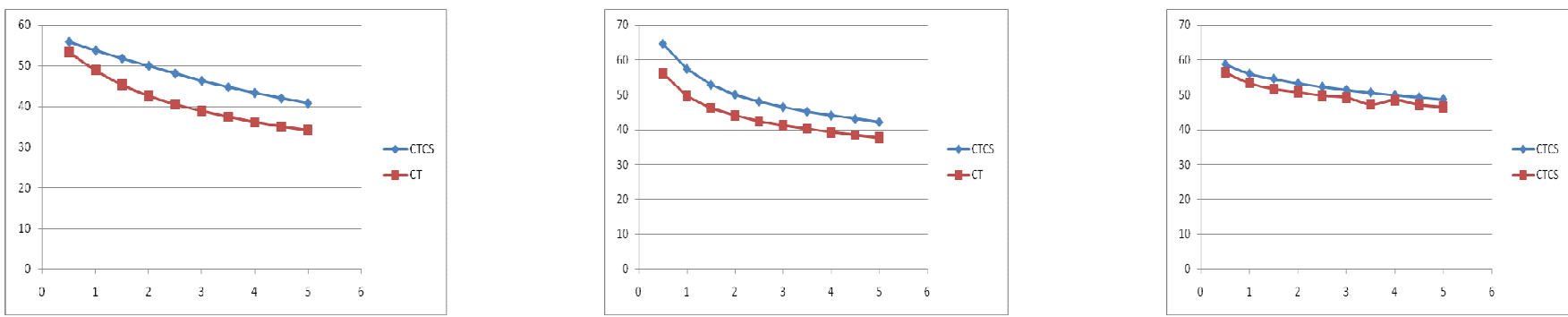

Fig.2 Comparison Chart of PSNR v/s variance of different denoising method of image size 256X256, 512x512 and 1024 x 1024 
Table 1: Comparison of PSNR, MSE and Time Elapsed of Different Denoised Image using Curvelet Transform with Cycle Spinning

\begin{tabular}{|c|c|c|c|c|c|c|c|c|c|}
\hline \multirow[b]{2}{*}{ Variance } & \multicolumn{3}{|c|}{$256 \times 256$ Image } & \multicolumn{3}{|c|}{512 x 512 image } & \multicolumn{3}{|c|}{$1024 \times 1024$ image } \\
\hline & MSE & $\begin{array}{c}\text { PSNR } \\
(\mathrm{db})\end{array}$ & $\begin{array}{c}\text { Time } \\
\text { elapsed } \\
\text { (in Sec) }\end{array}$ & MSE & $\begin{array}{c}\text { PSNR } \\
(\mathrm{db})\end{array}$ & $\begin{array}{c}\text { Time } \\
\text { elapsed } \\
\text { (in Sec) }\end{array}$ & MSE & $\begin{array}{c}\text { PSNR } \\
(\mathrm{db})\end{array}$ & $\begin{array}{c}\text { Time } \\
\text { elapsed } \\
\text { (in Sec) }\end{array}$ \\
\hline 0.5 & 0.1665 & 55.9155 & 2.5 & 0.023 & 64.5165 & 7.639 & 0.0866 & 58.7554 & 28.608 \\
\hline 1 & 0.2714 & 53.7947 & 2.56 & 0.121 & 57.302 & 7.522 & 0.1625 & 56.0234 & 34.993 \\
\hline 1.5 & 0.4291 & 51.8055 & 2.47 & 0.3399 & 52.8172 & 7.372 & 0.2289 & 54.5348 & 35.084 \\
\hline 2 & 0.6583 & 49.9601 & 2.4 & 0.6478 & 50.0165 & 7.295 & 0.3054 & 53.2821 & 36.702 \\
\hline 2.5 & 1.01 & 48.0837 & 2.47 & 1.0281 & 48.0105 & 8.134 & 0.3871 & 52.2523 & 38.022 \\
\hline 3 & 1.53 & 46.2808 & 2.67 & 1.477 & 46.4371 & 7.35 & 0.473 & 51.3821 & 36.833 \\
\hline 3.5 & 2.2147 & 44.677 & 2.44 & 1.9937 & 45.1342 & 7.365 & 0.5662 & 50.6011 & 37.1059 \\
\hline 4 & 3.0741 & 43.2537 & 2.52 & 2.5683 & 44.0343 & 7.396 & 0.6637 & 49.9114 & 36.464 \\
\hline 4.5 & 4.15 & 41.94 & 2.58 & 3.2318 & 43.0363 & 7.68 & 0.7699 & 49.2666 & 37.38 \\
\hline 5 & 5.49 & 40.7315 & 2.58 & 3.9702 & 42.1427 & 7.39 & 0.883 & 48.671 & 48.671 \\
\hline
\end{tabular}

Table 2: Comparison of PSNR, MSE and Time Elapsed of Different Denoised Image Using Curvelet Transform

\begin{tabular}{|c|c|c|c|c|c|c|c|c|c|}
\hline \multirow[b]{2}{*}{ Variance } & \multicolumn{3}{|c|}{$256 \times 256$ Image } & \multicolumn{3}{|c|}{$512 \times 512$ image } & \multicolumn{3}{|c|}{$1024 \times 1024$ image } \\
\hline & MSE & $\begin{array}{c}\text { PSNR } \\
(\mathrm{db})\end{array}$ & $\begin{array}{c}\text { Time } \\
\text { elapsed } \\
\text { (in } \\
\text { Sec) } \\
\end{array}$ & MSE & $\begin{array}{c}\text { PSNR } \\
(\mathrm{db})\end{array}$ & $\begin{array}{c}\text { Time } \\
\text { elapsed } \\
\text { (in } \\
\text { Sec) } \\
\end{array}$ & MSE & $\begin{array}{c}\text { PSNR } \\
(\mathrm{db})\end{array}$ & $\begin{array}{l}\text { Time } \\
\text { elapsed } \\
\text { (in Sec) }\end{array}$ \\
\hline 0.5 & 0.2963 & 53.414 & 5.25 & 0.1546 & 56.2375 & 8.693 & 0.1471 & 56.455 & 35.261 \\
\hline 1 & 0.8335 & 48.9217 & 3.83 & 0.717 & 49.5754 & 9.45 & 0.3014 & 53.3399 & 34.144 \\
\hline 1.5 & 1.973 & 45.3135 & 4.59 & 1.5796 & 46.1454 & 11.15 & 0.4438 & 51.6587 & 33.62 \\
\hline 2 & 3.5631 & 42.6125 & 6.33 & 2.5776 & 44.0186 & 11.09 & 0.549 & 50.7349 & 33.064 \\
\hline 2.5 & 5.8161 & 40.4841 & 5.5 & 3.7554 & 42.3483 & 10.12 & 0.6582 & 49.7475 & 35.2618 \\
\hline 3 & 8.4296 & 38.8727 & 5.53 & 4.9436 & 41.1903 & 10.11 & 0.786 & 49.1766 & 35.471 \\
\hline 3.5 & 11.6015 & 37.4283 & 3.82 & 6.1185 & 40.2644 & 9.88 & 0.9343 & 47.1787 & 31.679 \\
\hline 4 & 15.9402 & 36.1059 & 5.58 & 8.0073 & 39.0959 & 8.064 & 1.0995 & 48.4261 & 42.042 \\
\hline 4.5 & 20.4261 & 35.0289 & 4.63 & 9.5325 & 38.3387 & 9.275 & 1.2824 & 47.0507 & 33.75 \\
\hline 5 & 25.1746 & 34.1214 & 4.43 & 11.2393 & 37.6234 & 9.1056 & 1.4278 & 464053 & 32.214 \\
\hline
\end{tabular}

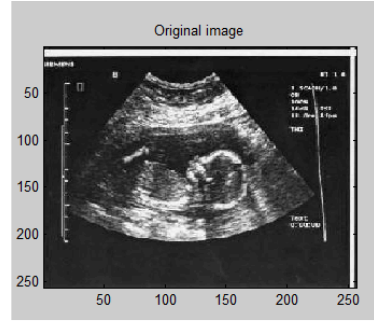

a)

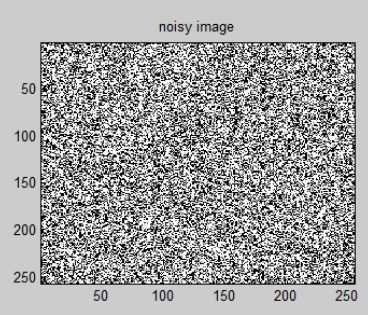

b)

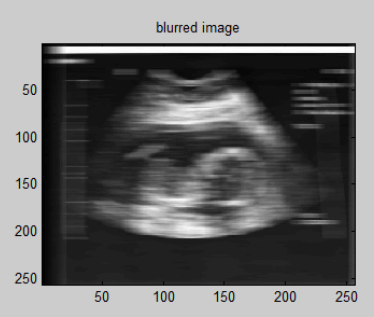

c) 


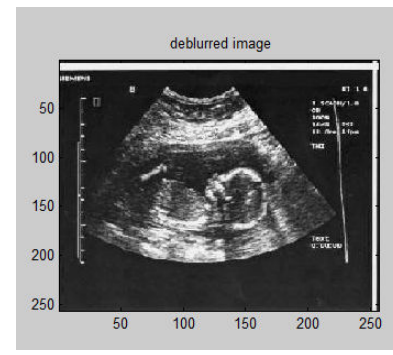

d)

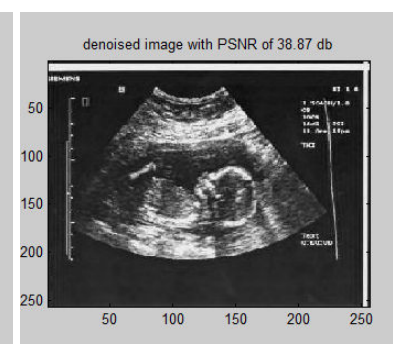

e)

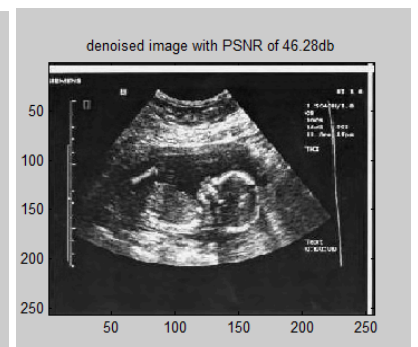

f)

Fig 3 a) Original image of size $256 \times 256$ b) Speckle noise is added with variance of 3.0 c) blurred image shows the subject move with the linear velocity of $10 \mathrm{~m} / \mathrm{sec}$ for $2 \mathrm{sec} \mathrm{d}$ ) degraded image is restored e,f) denoised image using curvelet transform and curvelet transform with cycle spinning with PSNR of $38.87 \mathrm{db}, 46.28 \mathrm{db}$

\section{CONCLUSION}

Diagnosis using ultrasound images is difficult because the speckle noise which hampers the prediction and the extraction of fine details from the image.In this paper we concentrate on the improvements of interpretation of ultrasound images by the impulse response of received echo pulses Then, we study the impulse response of small region and the "real" trace would be in $3 \mathrm{D}$, but we use its $2 \mathrm{D}$ projection and especially in $\mathrm{x}$ direction. This could be possible when the movement's range is not as wide in one direction as in the others. CT shows huge potential in image denoising application as a new multi-scale transform. Experimental results show that the CTCS proposed in this paper increases PSNR of reconstructed images at the same time keeping information in high frequency subbands better and improving visual performance especially for those images possessing detailed textures.

\section{REFERNCES}

[1] Ashish Thakur and R. S. Anand, "Feature Extraction in Ultrasound Medical Images" Ph.D. Thesis, IIT Roorkee, ID No Ph.D. 2006/AT/RSA/33, pp. 15-76.

[2] S.C Som, J. Opt. Soc. Am. 61 (1971) pp. 859.

[3] Coifman R R, Donoho D L. Translation invariant denoising. In Wavelets and Statistics, Springer Lecture Notes in Statistics 103, New York: Springer-Verlag, 1995, pp.125150 .

[4] J. Starck, E. Cand_es, D. Donoho, The curvelet transform for image denoising, IEEE Trans.Image Process., 11, 670-684 (2002).

[5] E. Cand_es, F. Guo, New multiscale transforms, minimum total variation synthesis: applications to edge- preserving image reconstruction, Signal Process., 82 (11),1519-1543 (2002).

[6] J. Starck, F. Murtagh, E. Candues, F. Murtagh, D. Donoho, Gray and color image contrast enhancement by the curvelet transform, IEEE Trans. Image Process., 12 (6), 706-717 (2003).

[7] J. Starck, E. Candues, D. Donoho, Astronomical image representation by the curvelet transform, Astronomy and Astrophysics, 398, 785-800 (2003).
[8] M. Choi, R. Kim, M. Nam, H. Kim, Fusion of multispectral and panchromatic satellite images using the curvelet transform, IEEE Geosci. Remote Sensing Lett., 2 (2), 13614 (2005)

[9] E. Candues, D. Donoho, New tight frames of curvelets and optimal representations of objects with piecewise singularities, Comm. Pure Appl. Math., 57, 219-266 (2004).

[10] J. Ma, A. Antoniadis, F.-X. Le Dimet, Curvelet-based multiscale detection and tracking for geophysical fuids, IEEE Trans. Geosci. Remote Sensing, 44 (12), 3626-3637 (2006).

[11] J. Ma, Deblurring using singular integrals and curvelet shrinkage, Physics Letters A, 368, 245-250 (2007).

[12] J. Ma, G. Plonka, Combined curvelet shrinkage and nonlinear anisotropic diffusion,IEEE Trans. Image Process., 16 (9), 2198-2206 (2007).

[13] G. Plonka, J. Ma, Nonlinear regularized reaction-diffusion filters for denoising of images with textures, IEEE Trans. Image Process., 17 (8), 1283-1294 (2008).

[14] J. Starck, M. Elad, D. Donoho, Image decomposition via the combination of sparse representation and a variational approach, IEEE Trans. Image Process., 14 (10), 1570-1582 (2005).

[15] J. Bobin, J. Starck J. Fadili, Y. Moudden, D. Donoho, Morphological component analysis: an adaptive thresholding strategy, IEEE Trans. Image Process., 16 (11), 2675-2681 (2007).

[16] B. Zhang, J. Fadili, J. Starck, Wavelets, ridgelets, and curvelets for Poisson noise removal, IEEE Trans. Image Process., 17 (7), 1093-1108 (2008).

[17] C. Zhang, L. Cheng, Z. Qiu, L. Cheng, Multipurpose Water marking based on multiscale curvelet transform, IEEE Trans. Inform. Forensics and Security, 3 (4), 611-619 (2008).

[18] L. Jiang, X. Feng, H. Yin, Structure and texture image inpainting using sparse representations and an iterative curvelet thresholding approach, Wavelets,Multiresolution and Inform. Process., 6 (5), 691-705 (2008). 
[19] T. Geback, P. Koumoutsakos, Edge detection in microscopy images using curvelets, BMC Bioinformatics, 10 (75),1471-2105-10-75 ( 2009).

[20] L. Tessens, A. Pizurica, A. Alecu, A. Munteanu, W. Philips, Context adaptive image denoising through modeling of curvelet domain statistics, J. Electronic Imaging, 17 (3), 03021:1-17(2008)

[21] J. Meunier and M. Betrand, "Ultrasonic texture motion analysis: Theory and simulation," IEEE Trans. Med. Imag., vol. 14,pp.-293-300, 1995.

[22] Akash Tayal, Komal Juneja, "Ultrasound Image Restoration of Blurring Due to Rectilinear motion" proceedings of Third National Conference on Mathematical Techniques: Emerging Paradigms for Electronics and IT Industries (MATEIT)),India, pp. 6.3.1-6.3.4,2010

[23] G. Beylkin, R. Coifman and V. Rokhlin. "Fast wavelet transforms and numerical algorithm",Comm. on Pure and Appl. Math.44 (1991), pp.-141-183

[24] G. Beylkin. "On the fast Fourier transform of functions with singularities" Appl. Comput Harmon.Anal., 2-4 (1995), 363-381.

[25] E. J. Cand'es. "Harmonic analysis of neural networks" Applied and Computational HarmonicAnalysis 6 (1999), 197-218.

[26] E. J. Cand'es and L. Demanet, "The curvelet representation of wave propagators is Optimally sparse" Pure Appl. Math., 58-11 (2005) 1472-1528.

[27] E. J. Cand'es and L. Demanet. "Curvelets and fast wave equation solvers" Technical report, California Institute of Technology, 2005

[28] Cand $\mu$ es E, Donoho D. Curvelets: A Surprisingly Effective Nonadaptive Representation of Objects with Edges.Curves and Surface, Nashville: Vanderbilt University Press, USA, 1999, pp.123-143

[29] Feng Peng, Mi Deling, Pan Yingjun, Wei Biao and Jin Wei, "Noise Removal Approach using Curvelet Transform", Opto- Electronic Engineering, 2005, 32(9), pp. 67-70

[30] M. Young, Candes E J, Demanet L, Donoho D L, et al. "Fast Discrete Curvelet Transforms", Applied and Computational Mathematics, California Institute of Technology, 2005.

[31] Liang Dong, Shen Min, Gao Qingwei, Bao Wenxia and $\mathrm{Qu}, \quad$ Lei, "A Method for Image De-noising Based on the Contourlet Transform Using Recursive Cycle Spinning", Chinese Journal of Electronics,2005, 33(11), pp. 20442046.

[32] L. Boubchir and M.J Fadili, "Multivariate Statistical Modeling of Images with the Curvelet Transform", in Proc. 8th International Conference on Signal Proc. And Its Applications, 2005, pp. 747-750.

[33] Nquyen Truong T, "Multiresolution Direction Filterbanks: Theory, Design, and Applications", IEEE Transactions on Signal Processing, 2005,53(101):3895-3905.

[34] Langis Ganon “ Wavelet Filtering of Speckle Noise-Some Numerical Result," Proceeding of the Conference Vision Interface 1999 , Trois-Riveres

[35] J. C. Bamber and C. Daft. Adaptive filtering for reduction of speckle in ultrasonic pulse echo images. Ultrasonics, pages 41-44, 1986. 